\title{
Hypofractionated postoperative helical tomotherapy in prostate cancer: a mono- institutional report of toxicity and clinical outcomes
}

This article was published in the following Dove Press journal: Cancer Management and Research

\section{Francesco Cuccial,2 \\ Gianluca Mortellaro ${ }^{2}$ \\ Vincenzo Serretta ${ }^{3}$ \\ Vito Valenti ${ }^{1,2}$ \\ Antonella Tripoli ${ }^{1,2}$ \\ Marina Gueci ${ }^{1,2}$ \\ Nicoletta Luca ${ }^{1,2}$ \\ Antonio Lo Casto 4 \\ Giuseppe Ferrera ${ }^{2}$ \\ 'Radiation Oncology School, University of Palermo, Palermo, Italy; ${ }^{2}$ Radiation Oncology, ARNAS-Civico Hospital, Palermo, Italy; ${ }^{3}$ Section of Urology, Department of Surgical Oncological and Oral Science, University of Palermo, Palermo, Italy; ${ }^{4}$ Radiation Oncology School, Section of Radiological Sciences, DIBIMED, Università degli Studi di Palermo. Palermo, Italy}

Purpose: This is a mono-institutional study of acute and late toxicities and early biochemical control of a retrospective series of 75 prostate cancer patients treated with moderate postoperative hypofractionation delivered by helical tomotherapy (HT).

Patients and methods: From April 2013 to June 2017, 75 patients received adjuvant ( $\mathrm{n}=37$ ) or salvage $(n=38)$ treatment, delivering to prostate bed a total dose of $63.8 \mathrm{~Gy}$ (equivalent dose in 2-Gy fractions $=67.4 \mathrm{~Gy}$ ) using $2.2 \mathrm{~Gy}$ fractions. Whole-pelvis irradiation was performed in $63 \%$ of cases (median dose, $49.3 \mathrm{~Gy}$; range, 48-55.1 Gy). Concurrent hormonal therapy was administered in 46\% of cases. Common Terminology Criteria for Adverse Events (version 4.0) was adopted for acute and late genitourinary $(\mathrm{GU})$ and gastrointestinal (GI) toxicity evaluations. Biochemical progression was defined as PSA level increase of $\geq 0.2$ or more above the postoperative radiotherapy (RT) nadir. Results: Acute GU toxicities were as follows: $\mathrm{G} 1$ in $46 \%$ and $\mathrm{G} 2$ in $4 \%$, detecting no $\mathrm{G} \geq 3$ events. For GI toxicity, we recorded G1 in 36\% and G2 in 18\%. With a median follow-up of 30 months (range, 12-58 months), we found late toxicity G2 GI in $6.6 \%$ and G $\geq 2$ GU in 5.3\%, including two patients who underwent surgical incontinence correction. Acute GI $\geq 2$ toxicity and diabetes were found to be predictive of late $\mathrm{GI} \geq 2$ toxicity ( $P=0.04$ and $P=0.0019)$. Actuarial 2 - and 3-year biochemical recurrence-free survivals were $88 \%$ and $73 \%$, respectively, for the entire population.

Conclusion: In our experience, moderate hypofractionated postoperative RT with HT was feasible and safe, with reports of low incidence of toxicity and promising biochemical control rates. Keywords: prostate neoplasm, radiotherapy, hypofractionation, adjuvant, salvage

\section{Introduction}

Prostate cancer (PC) is the most common cancer in European Union in men older than 70 years, with a higher incidence in Northern and Western Europe ( $>200$ cases per 100,000). ${ }^{1}$ In localized PC, radiation therapy has an important role in definitive or postoperative setting with or without androgen deprivation therapy (ADT). Three important randomized trials with long follow-up (SWOG 8794, EORTC 22911, and ARO 96-02) reported significant improvements in biochemical recurrence-free survival (bRFS) with the use of adjuvant radiotherapy compared to radical prostatectomy alone among patients with adverse pathological features. ${ }^{2-4}$

On the other hand, two of these randomized trials, reporting that more than $40 \%$ of patients addressed to observation after surgery will not have any recurrence after 10 years of follow-up, underline the potential risk of overtreating a subgroup of patients
Correspondence: Francesco Cuccia Radiation Oncology School, University of Palermo, Piazza Nicola Leotta, Palermo 4 - 90100 , Italy

Tel +393334393336

Fax +3909I 6664003

Email f.cuccia I@virgilio.it 
exposed to short- and long-term side effects without the evidence of a clear benefit in terms of disease control. ${ }^{5,6}$

In these patients, initial observation after radical prostatectomy may be the correct choice, keeping salvage radiotherapy (RT) as a useful option in case of biochemical relapse. ${ }^{7}$

Briganti et a ${ }^{8}$ recently developed a predictive nomogram to recognize patients for early salvage instead of adjuvant treatment.

In patients with adverse pathological features, therefore, few prospective multicenter randomized trials are currently ongoing and evaluating the timing of postoperative treatment (early vs deferred) and the duration of hormone therapy (none vs short-term vs long-term), aiming to clarify the contrasting evidence currently available from retrospective studies with insufficient follow-up or heterogeneous population. ${ }^{9,10}$

Several retrospective studies investigated the potential of dose escalation in the postoperative setting, confirming the positive correlation between higher doses and bRFS rates; ${ }^{11-15}$ however, the optimal dose still remains controversial. Based on the radiobiological properties of $\mathrm{PC}$, as a tumor more sensitive to higher doses per fraction, the growth of modern RT techniques lead to the current spread of moderate and extreme hypofractionated treatments for the nonsurgical patient. ${ }^{16}$

However, in contrast to the definitive setting, few data are available on hypofractionated postoperative RT.

Herein, we report our preliminary results of postprostatectomy hypofractionation schedule using helical tomotherapy (HT; Accuray, Inc., Sunnyvale, CA, USA), which associates intensity modulated radiotherapy (IMRT) delivered with a helical system with an image guidance system based on daily megavoltage computed tomography (CT) scan.

\section{Materials and methods}

This is a retrospective mono-institutional analysis of 75 patients with histologically proven adenocarcinoma of the prostate undergoing moderate postoperative hypofractionated RT delivered by HT.

Adjuvant treatment, given within 6 months after surgery with PSA $\leq 0.2 \mathrm{ng} / \mathrm{mL}$, was performed in the presence of adverse pathological features (extracapsular extension, invasion of seminal vesicles, positive margins, and lymph nodal involvement). Salvage therapy was delivered 6 months after surgery with PSA $\geq 0.2 \mathrm{ng} / \mathrm{mL}$.

ADT was administered, according to the discretion of the referring urologist, in patients with seminal vesicle invasion, nodal involvement, Gleason Score $>7$, or PSA $>20 \mathrm{ng} / \mathrm{mL}$.

This study was approved by the Steering Ethical Committee Palermo 2. Written informed consent was obtained from all patients to review their medical records, as required by the institutional review board. All patients' data are confidential and anonymously recorded.

The primary aim was to report the acute and late toxicities, and the secondary endpoint was to evaluate early biochemical control.

\section{Radiation planning and treatment}

All patients underwent a $2.5 \mathrm{~mm}$ thickness slice CT simulation. Planning CT and treatment were performed with a full bladder ( $500 \mathrm{~mL}$ of water was given 30 minutes before the procedure) and empty rectum in a supine position with flexed legs positioned in knee and ankle devices. As organs at risk (OARs), we delineated bladder, rectum, small bowel, intestinal cavity, and femoral heads. Prostate bed and pelvic lymph nodes clinical target volumes (CTV1-CTV2) were delineated using Radiation Therapy Oncology Group consensus guidelines. ${ }^{17,18}$ The planning target volume (PTV) 1 (PTV1) was obtained adding to CTV1 a margin of $5 \mathrm{~mm}$ in all directions. The CTV2 was expanded by 5-7 mm to generate PTV2. Following American Urological Association/American Society for Radiation Oncology guidelines, ${ }^{19}$ recommending a minimum doses of 64 and $65 \mathrm{~Gy}_{2}$ for adjuvant and salvage RTs, respectively, and assuming an $\alpha / \beta=1.5$ Gy for PC, we adopted 2.2 Gy fractions to deliver a total dose of $63.8 \mathrm{~Gy}$ (equivalent dose in 2-Gy fractions $\left[\mathrm{EQD}_{2}\right]=67.4 \mathrm{~Gy}$ ) to prostate bed and a median dose of $49.3 \mathrm{~Gy}\left(\mathrm{EQD}_{2}=45.1 \mathrm{~Gy}\right.$; range, $\left.48-55.1 \mathrm{~Gy}\right)$ in conventional fractionation $(1.7-1.9 \mathrm{~Gy} / \mathrm{fx})$ to the pelvic lymph nodes using simultaneous integrated boost (SIB) technique. Pelvic lymph nodes irradiation was planned in patients with the following pathological features: $\mathrm{pN}+$ and/or lymph nodal dissection $<10$ nodes and/or Gleason Score $>8$.

The dosimetric goal was to cover $95 \%$ of PTVs with at least $95 \%$ of the prescribed dose; OARs planning constraints were as follows: V $56 \mathrm{~Gy} \leq 35 \%$ and $\mathrm{V} 60 \mathrm{~Gy} \leq 25 \%$ for rectum, and V55Gy $\leq 50 \%$ and V60Gy $\leq 30 \%-35 \%$ for bladder. For the intestinal cavity, the dose was reduced as low as possible.

Inverse IMRT planning was performed using the Tomotherapy (Accuray, Inc.) planning software. Our image guided radiotherapy protocol consists of a daily megavoltage computed tomography (MVCT) considering the intrafraction variability of OARs to check setup accuracy and to assess appropriate bladder filling and rectal emptying.

\section{Toxicity evaluation}

The acute and late genitourinary (GU) and gastrointestinal (GI) radiation-related toxicities were scored according to the Common Terminology Criteria for Adverse Events (CTCAE, 
version 4.0). Biochemical progression was defined as PSA level increase of $\geq 0.2$ or more above the postoperative RT nadir.

Clinical evaluation of acute toxicity was performed weekly during the treatment and then at 40 and 90 days after the end of RT. Afterward, we evaluated the late events every 3-6 months for the first 2 years and then at biannual and annual intervals.

\section{Statistical analyses}

Frequencies and percentages are reported for GU and GI toxicities; medians and ranges were calculated for continuous variables. Statistical analyses were performed with chisquared tests assuming $P \leq 0.05$ as statistically significant. Survival curves were generated with Kaplan-Meier method. All statistical analyses were carried out using MedCalc statistical software package, version 18.5 (Mariakerke, Belgium).

\section{Results}

From April 2013 to June 2017, 75 patients with median age of 68 years (range, 54-84 years) were treated with hypofractionated radiation therapy after prostatectomy. Patients' characteristics are summarized in Table 1. Adjuvant treatment was performed in $37(49 \%)$ patients and salvage therapy in 38 (51\%) patients. ADT was administered in 34 (46\%) patients.

A total dose of $63.8 \mathrm{~Gy}\left(\mathrm{EQD}_{2}=67.4 \mathrm{~Gy}\right)$ to prostate bed was delivered. Pelvic lymph nodes irradiation with a median dose of 49.3 Gy (range, 48-55.1 Gy) in conventional fractionation was administered in $47(63 \%)$ patients.

All 75 patients completed the planned treatment without any interruption, with good tolerance.

Acute GU toxicities were as follows: G1 in 35 (46\%) and $\mathrm{G} 2$ in three (4\%) patients, no $\mathrm{G} \geq 3$ events were detected; the main symptom reported was urinary tract pain, which occurred in 18 (24\%) patients. For GI toxicity, we recorded G1 in $36 \%$ of patients $(n=27)$ and G2 in $18 \%$ of patients $(n=14)$. Most frequent GI adverse event was diarrhea in 19 (25\%) cases. Table 2 presents specific acute symptoms reported according to CTCAE, version 4.0.

After a median follow-up of 30 months (range, 12-58 years), we detected G2 GI late toxicity in five (6.6\%) cases; no $\mathrm{G} 3$ toxicity was observed, and $\mathrm{G} \geq 2 \mathrm{GU}$ late toxicity was observed in four (5.3\%) patients, consisting of two G2 late events and two G3 patients who underwent surgical incontinence correction after 24 and 36 months, respectively (Figure 1).

Also, dosimetric parameters, bladder and rectum V45 and V60, were not related to acute and late toxicity patterns, respectively. Only acute GI G2 toxicity and diabetes were
Table I Patients' characteristics

\begin{tabular}{|c|c|}
\hline Characteristics & Median (range) or $n(\%)$ \\
\hline Age (years) & $68(54-84)$ \\
\hline Follow-up (months) & $30(12-58)$ \\
\hline \multicolumn{2}{|l|}{ Diabetes } \\
\hline Yes & $17(23)$ \\
\hline No & $58(77)$ \\
\hline PSA pre-RT (ng/mL) & $0.19(0-7.03)$ \\
\hline \multicolumn{2}{|l|}{ Gleason Score } \\
\hline$\leq 7$ & $58(77)$ \\
\hline$\geq 8$ & $17(23)$ \\
\hline \multicolumn{2}{|l|}{$\mathrm{pT}$} \\
\hline $\mathrm{pT} 2 \mathrm{a}$ & I (2) \\
\hline pT2b & $6(8)$ \\
\hline pT2c & $17(23)$ \\
\hline pT3a & $22(29)$ \\
\hline $\mathrm{pT} 3 \mathrm{~b}$ & $27(36)$ \\
\hline pT4 & $\mathrm{I}(2)$ \\
\hline \multicolumn{2}{|l|}{$\mathrm{pN}+$} \\
\hline No & $63(84)$ \\
\hline Yes & $12(16)$ \\
\hline \multicolumn{2}{|l|}{ Surgical margins } \\
\hline Negative & $44(59)$ \\
\hline Positive & $3 I(4 I)$ \\
\hline \multicolumn{2}{|l|}{ RT } \\
\hline Adjuvant & $37(49)$ \\
\hline Salvage & $38(5 \mathrm{I})$ \\
\hline \multicolumn{2}{|l|}{ Pelvic nodal RT } \\
\hline No & $28(37)$ \\
\hline Yes & $47(63)$ \\
\hline \multicolumn{2}{|l|}{$\mathrm{RT}+\mathrm{ADT}$} \\
\hline No & $4 I(54)$ \\
\hline Yes & $34(46)$ \\
\hline
\end{tabular}

Abbreviations: ADT, androgen deprivation therapy; RT, radiotherapy.

Table 2 Acute $\mathrm{Gl}$ and $\mathrm{GU}$ adverse events according to the CTCAE version 4.0 scale

\begin{tabular}{|l|l|l|l|}
\hline GI symptoms & Grade I & Grade 2 & Grade 3 \\
\hline Tenesmus & $\mathrm{II}$ & 8 & - \\
\hline Diarrhea & $\mathrm{I} 5$ & 4 & - \\
\hline Rectal bleeding & - & $\mathrm{I}$ & \\
\hline Hemorrhoids & $\mathrm{I}$ & $\mathrm{I}$ & - \\
\hline GU symptoms & Grade I & Grade 2 & Grade 3 \\
\hline Urinary tract pain & $\mathbf{I 6}$ & 2 & - \\
\hline Urinary frequency & 8 & - & - \\
\hline Incontinence worsening & $\mathrm{I}$ & - & - \\
\hline Urgency & 7 & $\mathrm{I}$ & - \\
\hline
\end{tabular}

Abbreviations: CTCAE, Common Terminology Criteria for Adverse Events; GI, gastrointestinal; GU, gastrourinary.

found to be predictive of late GI G2 toxicity ( $P=0.04$ and $P=0.0019$, respectively).

In a subgroup analysis, a higher incidence of acute GI G2 toxicity in patients who underwent whole-pelvis irradiation was observed, detecting 12 cases $(25 \%)$ vs two events 
$(7 \%)$ in the prostate bed alone subgroup. Actuarial 2- and 3 -year bRFSs were $88 \%$ and $73 \%$, respectively, for the entire population (Figure 2).

We failed to find any significant correlation among pelvic RT $(P=0.25)$, adjuvant or salvage intent $(P=0.28)$, hormone therapy $(P=0.32)$, and bRFS rates.

At the time of the analysis, all patients are alive except one who died because of cerebrovascular disease.

\section{Discussion}

Our clinical experience with postprostatectomy moderate hypofractionation using HT confirmed that, with this delivery technique, toxicities are quite low and similar to those observed in other hypofractionation studies in this setting. ${ }^{20}$
The use of hypofractionation in PC comes from the well-known evidence of the very low $\alpha / \beta$ ratio of the tumor that leads to improved tumor control using higher doses per fraction..$^{21,22}$

As these evidences are supported by several randomized Phase III trials for the definitive patient, ${ }^{23-25}$ few studies in literature evaluated hypo-RT in the postoperative setting, reporting favorable toxicity profiles with very low rates of $\mathrm{G}>2$ toxicity (Table 3 ). ${ }^{26-35}$

Fersino et a ${ }^{30}$ reported only one case of acute G3 urinary toxicity in their series of 125 patients (64 adjuvant and 61 salvage) treated with hypofractionated volumetric modulated arc therapy (VMAT), and at the time of final assessment, they collected no $\mathrm{G}>2$ late toxicity.
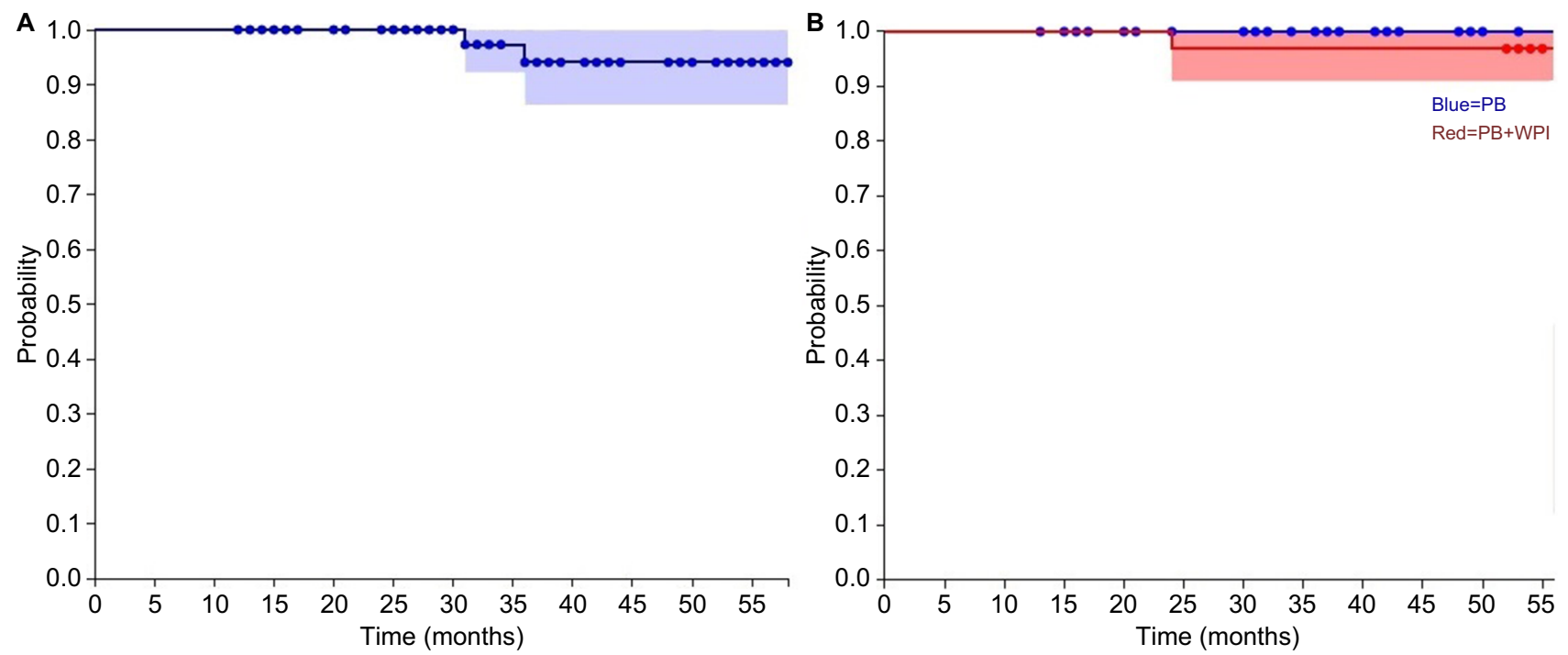

Figure I G3 toxicity free-survival curves for the entire population (A) and according to radiotherapy volumes (B) (prostate bed only vs prostate bed and whole-pelvis irradiation)

Abbreviations: PB, prostate bed; WPI, whole pelvis irradiation.
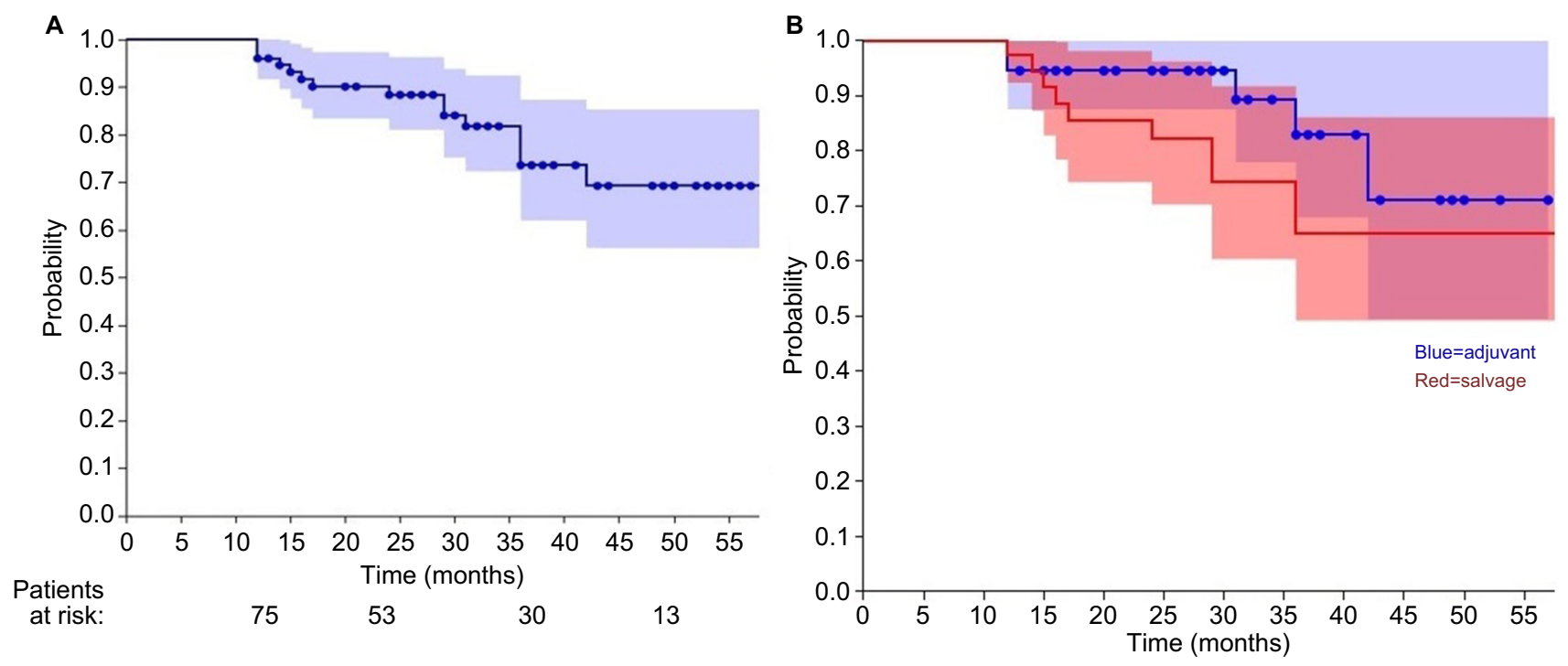

Figure 2 Biochemical relapse-free survival curves for the entire population (A) and stratified for adjuvant and salvage treatment (B). 
Similar results were observed in the study by Massaccesi et $\mathrm{al}^{27}$ in their prospective trial of postoperative IMRT to the whole pelvis (45 Gy/25fx) plus a SIB of $62.5 \mathrm{~Gy} / 25 \mathrm{fx}$ delivered to the prostate fossa, observing no $\mathrm{G}>2$ acute toxicity in their series of 49 patients.

More recently, the same RT schedule was evaluated by Macchia et $\mathrm{al}^{29}$ who published data about 124 patients using SIB-IMRT technique with concurrent hormonal therapy; with a median follow-up of 30 months, the authors observed one case of acute G4 urinary adverse event, and 5-year GI and GU toxicity rates of $1.1 \%$ and $7.3 \%$, respectively; therefore, they also collected very promising results in terms of biochemical control, with 2- and 3-year bRFS rates of $96.5 \%$ and $91.1 \%$, respectively, remarking the role of IMRT in improving the radiobiological effectiveness of treatment and assuring an excellent OARs sparing.

Actually, data on the use of HT in the hypofractionation postoperative setting are limited. Katayama et $\mathrm{al}^{33}$ in their series of 40 patients treated in the postprostatectomy setting with 54 Gy in 18 fractions delivered to prostate bed reported excellent data in terms of acute toxicity, with no G3 adverse event observed, despite a report on late side effects is currently lacking.

Kruser et al $^{34}$ reported only one G3 GU acute toxicity event, and no G3 late side effect in their series of 108 patients (59 with tomotherapy and 49 with linear accelerator-based IMRT) who underwent a hypofractionated schedule of 65 Gy/2.5 Gy/fx. Similarly, Barra et $\mathrm{al}^{35}$ published their study on 64 patients treated with the same schedule, collecting only G1 acute GU and GI toxicities and reporting late G3 GU adverse events only in $3.3 \%$ of cases.

Also in our population, the use of HT guaranteed an acceptable tolerability, in agreement with other hypofractionation experiences in this setting.

The interpretation of these findings in the light of other published data is challenging due to differences in treatment schedules and inhomogeneity of treated population. ${ }^{36}$

Similar to these and other IMRT studies, in our series, there was no association between GU toxicity and clinical or dosimetric parameters, although observing GU side effects being slightly more severe than GI ones.

Delineation of target volumes may have contributed to our side effects patterns, as we adopted Radiation Therapy Oncology Group guidelines. Compared to EORTC and FROGG guidelines, Radiation Therapy Oncology Group delineates a volume of prostate bed CTV encompassing a larger volume of bladder, maintaining a significantly lower exposure of rectum and mesorectal fascia. ${ }^{37,38}$ As reported by Ko et al, ${ }^{39}$ the vesico-urethral anastomosis represents the most frequent site of relapse, and it must be encompassed with posterior bladder wall in prostate bed CTV, leading to high exposure of normal bladder tissue, with an increased risk both in terms of frequency and severity of acute and late GU toxicities.

With regard to GI side effects, in our series, no G3 acute or late toxicity was observed as we reported only $\mathrm{G} 2$ adverse events in $18 \%$ and $6 \%$, respectively, and we found diabetes and acute GI toxicity to be predictive of late GI toxicity.

Despite the real benefit in terms of clinical outcomes is still under debate even for the definitive setting, ${ }^{40,41}$ we decided to treat pelvic lymph nodes for patients at risk of nodal involvement still reporting a higher incidence, yet not statistically significant, of GI toxicity, compared to patients not addressed to whole-pelvis irradiation $(P=0.06)$. This may be explained by the most frequent adoption of a safe schedule of $49.3 \mathrm{~Gy}$ in conventional fractionation that we mainly applied in pN0 patients but positive for other histopathological risk factors. Longobardi et $\mathrm{al}^{42}$ reported an excellent profile of toxicity both in definitive and in postoperative setting, in their series of 178 patients who underwent whole-pelvis bed irradiation+SIB to prostate/prostate with HT.

Our favorable toxicity rates can also be related to our prescription dose. Albeit the optimal dose for prostate bed still remains controversial, ${ }^{7,31,36}$ compared to other studies on hypofractionated postprostatectomy RT, we adopted a more conservative $\mathrm{EQD}_{2}$ prescription $\left(67.4 \mathrm{~Gy}_{2}\right)$, which allowed to reach a curative dose, maintaining a low probability of toxicity compared to the $2.5 \mathrm{~Gy} / \mathrm{fx}$ schedule, which is the most reported in literature (Table 3 ).

Indeed, Cozzarini et $\mathrm{al}^{31}$ investigated late toxicity patterns in a mono-institutional cohort of 247 patients treated with moderate hypofractionated HT, reporting a higher incidence of G3 urinary toxicity in the $>2 \mathrm{~Gy} / \mathrm{fx}$ subgroup. This is one of the largest series about late sequelae in postoperative prostate hypofractionation, with a median follow-up of 69 months and G3-4 late urinary incidence of 16.5\%. Keeping in mind the different schedules adopted in this series $(65.8$ Gy/2.35 Gy/fx; 71.4 Gy/2.5-2.6 Gy/fx; and 58 Gy/2.9 Gy/ fx). The authors explained these findings to be due to the negative effect of surgery, which does not allow the potential of bladder urothelium recovery from radiation-induced damage, resulting in a higher risk of urinary late toxicity when doses per fraction $>2.55$ Gy are used..$^{43}$

Also different from the study by Cozzarini et al, we used a tighter margin of $0.5 \mathrm{~cm}$ from CTV to PTV, which is considered the minimum recommended when daily online image guidance is adopted. ${ }^{44}$

As in conventional fractionation, the use of image guided radiotherapy represents an established tool to lower 


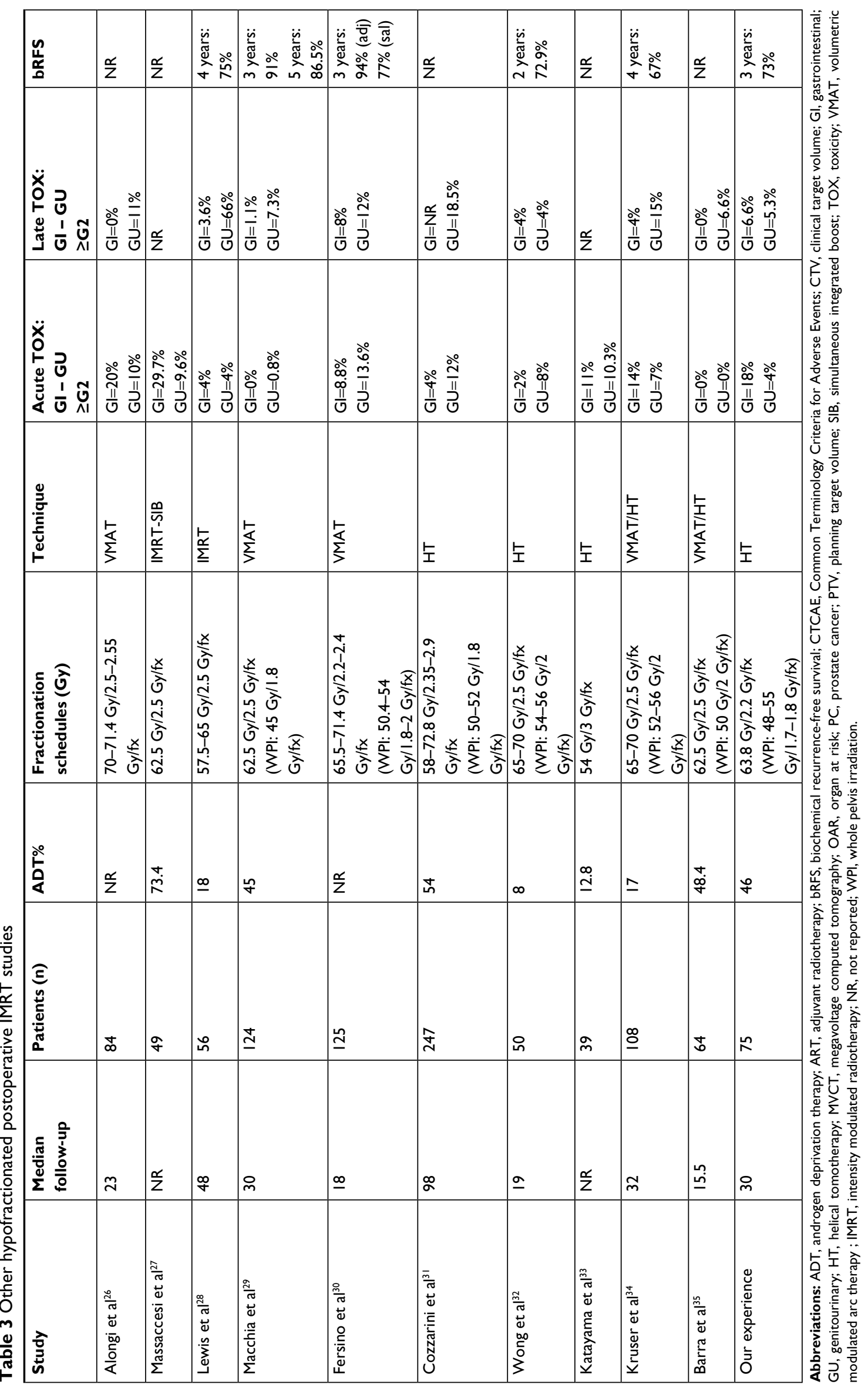


toxicity rates in the postoperative setting as it allows a more precise coverage of the target, minimizing OARs exposure, with a remarkable improvement of the therapeutic ratio. ${ }^{45,46}$

Consistent with these findings, at the time of the final analysis, the impact of our schedule in terms of biochemical control reflects in a 3 -year bRFS of $73 \%$ rate in agreement with literature data ranging from $72.9 \%$ to $85.5 \%$ at $2-3$ years ${ }^{20}$, confirming the efficacy of our treatment schedule.

The important limitations of our study are the relatively low number of patients and short follow-up. Moreover, we lack a well-designed quality of life study.

\section{Conclusion}

Our clinical experience with moderate postoperative hypofractionation using HT confirms low toxicity rates. In addition, we found encouraging preliminary data on biochemical control. Nevertheless, a longer follow-up is required for definitive assessment of clinical outcome.

\section{Author contributions}

All authors contributed toward data analysis, drafting and critically revising the paper and agree to be accountable for all aspects of the work.

\section{Disclosure}

The authors report no conflicts of interest in this work.

\section{References}

1. Arnold M, Karim-Kos HE, Coebergh JW, et al. Recent trends in incidence of five common cancers in 26 European countries since 1988: Analysis of the European Cancer Observatory. Eur J Cancer. 2015;51(9):1164-1187.

2. Thompson IM, Tangen CM, Paradelo J, et al. Adjuvant radiotherapy for pathological T3N0M0 prostate cancer significantly reduces risk of metastases and improves survival: long-term follow up of a randomized clinical trial. J Urol. 2009;181(3):956-962.

3. Bolla M, van Poppel H, Tombal B, et al. Postoperative radiotherapy after radical prostatectomy for high-risk prostate cancer: long-term results of a randomised controlled trial (EORTC trial 22911). Lancet. 2012;380(9858):2018-2027.

4. Wiegel T, Bartkowiak D, Bottke D, et al. Adjuvant radiotherapy versus wait-and-see after radical prostatectomy: 10-year follow-up of the ARO 96-02/AUO AP 09/95 trial. Eur Urol. 2014;66(2):243-250.

5. Arcangeli S, Ramella S, de Bari B, Franco P, Alongi F, D'Angelillo RM. A cast of shadow on adjuvant radiotherapy for prostate cancer: A critical review based on a methodological perspective. Crit Rev Oncol Hematol. 2016;97:322-327.

6. Sundaresan P, Turner S, Kneebone A, Pearse M, Fraser-Browne C, Woo HH. Do screening trial recruitment logs accurately reflect the eligibility criteria of a given clinical trial? Early lessons from the RAVES 0803 trial. Clin Oncol. 2014;26(6):348-352.

7. Gandaglia G, Briganti A, Clarke N, et al. Adjuvant and salvage radiotherapy after radical prostatectomy in prostate cancer patients. Eur Urol. 2017;72(5):689-709.
8. Briganti A, Karnes RJ, Joniau S, et al. Prediction of outcome following early salvage radiotherapy among patients with biochemical recurrence after radical prostatectomy. Eur Urol. 2014;66(3):479-486.

9. RADICALS [homepage on the Internet]. Radiotherapy and androgen deprivation in combination after local surgery. Available from: http:// www.radicals-trial.org/. Accessed September 27, 2018.

10. ANZCTR [homepage on the Internet]. Radiotherapy following radical prostatectomy - Adjuvant Versus Early Salvage. Available from: https:// www.anzctr.org.au/Trial/Registration/TrialReview.aspx?id=83910. Accessed September 27, 2018

11. Bellavita R, Massetti M, Abraha I, et al. Conformal postoperative radiotherapy in patients with positive resection margins and/or pT3-4 prostate adenocarcinoma. Int J Radiat Oncol Biol Phys. 2012;84(3):e299-e304.

12. Ost P, Fonteyne V, Villeirs G, Lumen N, Oosterlinck W, De Meerleer G. Adjuvant high-dose intensity-modulated radiotherapy after radical prostatectomy for prostate cancer: clinical results in 104 patients. Eur Urol. 2009;56(4):669-675.

13. Cozzarini C, Montorsi F, Fiorino C, et al. Need for high radiation dose (>or=70 gy) in early postoperative irradiation after radical prostatectomy: a single-institution analysis of 334 high-risk, node-negative patients. Int J Radiat Oncol Biol Phys. 2009;75(4):966-974.

14. Zilli T, Jorcano S, Peguret N, et al. Dose-adapted salvage radiotherapy after radical prostatectomy based on an erMRI target definition model: toxicity analysis. Acta Oncol. 2014;53(1):96-102.

15. Ghadjar P, Hayoz S, Bernhard J, et al. Acute toxicity and quality of life after dose-intensified salvage radiation therapy for biochemically recurrent prostate cancer after prostatectomy: first results of the randomized trial SAKK 09/10. J Clin Oncol. 2015;33(35):4158-4166.

16. Benjamin LC, Tree AC, Dearnaley DP. The Role of Hypofractionated Radiotherapy in Prostate Cancer. Curr Oncol Rep. 2017;19(4):30.

17. Michalski JM, Lawton C, El Naqa I, et al. Development of RTOG consensus guidelines for the definition of the clinical target volume for postoperative conformal radiation therapy for prostate cancer. Int $J$ Radiat Oncol Biol Phys. 2010;76(2):361-368.

18. Lawton CA, Michalski J, El-Naqa I, et al. RTOG GU Radiation oncology specialists reach consensus on pelvic lymph node volumes for high-risk prostate cancer. Int J Radiat Oncol Biol Phys. 2009;74(2):383-387.

19. Thompson IM, Valicenti RK, Albertsen P, et al. Adjuvant and salvage radiotherapy after prostatectomy: AUA/ASTRO Guideline. $J$ Urol. 2013;190(2):441-449.

20. Siepe G, Buwenge M, Nguyen NP, et al. Postoperative Hypofractionated radiation therapy in prostate carcinoma: a systematic review. Anticancer Res. 2018;38(3):1221-1230.

21. Dasu A, Toma-Dasu I. Prostate alpha/beta revisited - an analysis of clinical results from 14168 patients. Acta Oncol. 2012;51(8):963-974.

22. Brenner DJ, Martinez AA, Edmundson GK, Mitchell C, Thames HD, Armour EP. Direct evidence that prostate tumors show high sensitivity to fractionation (low alpha/beta ratio), similar to late-responding normal tissue. Int J Radiat Oncol Biol Phys. 2002;52(1):6-13.

23. Incrocci L, Wortel RC, Alemayehu WG, et al. Hypofractionated versus conventionally fractionated radiotherapy for patients with localised prostate cancer (HYPRO): final efficacy results from a randomised, multicentre, open-label, phase 3 trial. Lancet Oncol. 2016;17(8):1061-1069.

24. Lee WR, Dignam JJ, Amin MB, et al. Randomized Phase III noninferiority study comparing two radiotherapy fractionation schedules in patients with low-risk prostate cancer. J Clin Oncol. 2016;34(20):2325-2332.

25. Arcangeli G, Saracino B, Arcangeli S, et al. Moderate Hypofractionation in high-risk, organ-confined prostate cancer: final results of a Phase III randomized trial. J Clin Oncol. 2017;35(17):1891-1897.

26. Alongi F, Cozzi L, Fogliata A, et al. Hypofractionation with VMAT versus 3DCRT in post-operative patients with prostate cancer. Anticancer Res. 2013;33(10):4537-4543.

27. Massaccesi M, Cilla S, Deodato F, et al. Hypofractionated intensitymodulated radiotherapy with simultaneous integrated boost after radical prostatectomy: preliminary results of a phase II trial. Anticancer Res. 2013;33(6):2785-2789. 
28. Lewis SL, Patel P, Song H, et al. Image guided Hypofractionated Postprostatectomy intensity modulated radiation therapy for prostate cancer. Int J Radiat Oncol Biol Phys. 2016;94(3):605-611.

29. Macchia G, Siepe G, Capocaccia I, et al. Hypofractionated postoperative IMRT in prostate carcinoma: a Phase I/II Study. Anticancer Res. 2017;37(10):5821-5828.

30. Fersino S, Tebano U, Mazzola R, et al. Moderate Hypofractionated Postprostatectomy Volumetric Modulated Arc Therapy With Daily Image Guidance (VMAT-IGRT): A Mono-institutional Report on Feasibility and Acute Toxicity. Clin Genitourin Cancer. 2017;15(4):e667-e673.

31. Cozzarini C, Fiorino C, Deantoni C, et al. Higher-than-expected severe (Grade 3-4) late urinary toxicity after postprostatectomy hypofractionated radiotherapy: a single-institution analysis of 1176 patients. Eur Urol. 2014;66(6):1024-1030.

32. Wong GW, Palazzi-Churas KL, Jarrard DF, et al. Salvage hypofractionated radiotherapy for biochemically recurrent prostate cancer after radical prostatectomy. Int J Radiat Oncol Biol Phys. 2008;70(2):449-455.

33. Katayama S, Striecker T, Kessel K, et al. Hypofractionated IMRT of the prostate bed after radical prostatectomy: acute toxicity in the PRIAMOS-1 trial. Int J Radiat Oncol Biol Phys. 2014;90(4):926-933.

34. Kruser TJ, Jarrard DF, Graf AK, et al. Early hypofractionated salvage radiotherapy for postprostatectomy biochemical recurrence. Cancer. 2011;117(12):2629-2636.

35. Barra S, Belgioia L, Marcenaro M, et al. Moderate hypofractionated radiotherapy after prostatectomy for cancer patients: toxicity and clinical outcome. Cancer Manag Res. 2018;10:473-480. eCollection 2018.

36. Picardi C, Perret I, Miralbell R, Zilli T. Hypofractionated radiotherapy for prostate cancer in the postoperative setting: What is the evidence so far? Cancer Treat Rev. 2018;62:91-96.

37. Latorzeff I, Sargos P, Loos G, Supiot S, Guerif S, Carrie C. Delineation of the prostate bed: The "Invisible Target" is still an issue? Front Oncol. 2017;7:108. eCollection 2017.
38. Silverman JM, Krebs TL. MR imaging evaluation with a transrectal surface coil of local recurrence of prostatic cancer in men who have undergone radical prostatectomy. AJR Am J Roentgenol. 1997;168(2):379-385.

39. Ko EC, Michaud AL, Valicenti RK. Postoperative radiation after radical prostatectomy. Semin Radiat Oncol. 2017;27(1):50-66.

40. Spiotto MT, Hancock SL, King CR. Radiotherapy after prostatectomy: improved biochemical relapse-free survival with whole pelvic compared with prostate bed only for high-risk patients. Int J Radiat Oncol Biol Phys. 2007;69(1):54-61.

41. Pommier P, Chabaud S, Lagrange JL, et al. Is There a Role for Pelvic Irradiation in Localized Prostate Adenocarcinoma? Update of the Long-Term Survival Results of the GETUG-01 Randomized Study. Int J Radiat Oncol Biol Phys. 2016;96(4):759-769.

42. Longobardi B, Berardi G, Fiorino C, et al. Anatomical and clinical predictors of acute bowel toxicity in whole pelvis irradiation for prostate cancer with Tomotherapy. Radiother Oncol. 2011;101(3):460-464.

43. Fiorino C, Cozzarini C, Rancati T, et al. Modelling the impact of fractionation on late urinary toxicity after postprostatectomy radiation therapy. Int J Radiat Oncol Biol Phys. 2014;90(5):1250-1257.

44. Poortmans P, Bossi A, Vandeputte K, et al. Guidelines for target volume definition in post-operative radiotherapy for prostate cancer, on behalf of the EORTC Radiation Oncology Group. Radiother Oncol. 2007;84(2):121-127.

45. Nath SK, Sandhu AP, Rose BS, et al. Toxicity analysis of postoperative image-guided intensity-modulated radiotherapy for prostate cancer. Int J Radiat Oncol Biol Phys. 2010;78(2):435-441.

46. Azelie C, Gauthier M, Mirjolet C, et al. Exclusive image guided IMRT vs. radical prostatectomy followed by postoperative IMRT for localized prostate cancer: a matched-pair analysis based on risk-groups. Radiat Oncol. 2012;7:158.
Cancer Management and Research

\section{Publish your work in this journal}

Cancer Management and Research is an international, peer-reviewed open access journal focusing on cancer research and the optimal use of preventative and integrated treatment interventions to achieve improved outcomes, enhanced survival and quality of life for the cancer patient. The manuscript management system is completely online and includes

\section{Dovepress}

a very quick and fair peer-review system, which is all easy to use. Visit http://www.dovepress.com/testimonials.php to read real quotes from published authors. 